\title{
Electrospinning technique in the nano-photocatalyst research
}

\author{
Cong-Ju Li, ${ }^{12^{*}}$ Guo-Rong $X u^{1}$
}

${ }^{1 *}$ College of Material Science and Engineering, Beijing Institute of Fashion Technology, Beijing 100029, P. R.China; tel.: +86 0106428 8192; fax: +86 0106428 8192; e-mail: congjuli@gmail.com

${ }^{2}$ Beijing Key Laboratory of Clothing Materials R \& D and Assessment, Beijing 100029, P. R. China.

(Received: 16 March, 2010; published: 02 March, 2011)

\begin{abstract}
Photocatalysts have attracted much scientific interest stemming from their high catalytic efficiency and environmental friendly properties. However, due to the development of nanosicence and nanotechnology, nano-photocatalysts have attracted more and more attention. Generally speaking, nano-photocatalyst indicates photocatalytic materials in nano size, including nanoparticles, nanofibers, nanowires, and so on. Electrospinning, used as a simple method to fabricate nanomaterials, is playing a very important role on nano-photocatalyst research, both on the production of novel nano-photocatalysts and on the study of innovative fabrication methods. The applications of electrospinning on nano-photocatalyst research are reviewed with an emphasis on fabrication of nano-photocatalysts and on the strategies that have been tried to improve nano-photocatalysts performance.

Keywords: electrospinning, photocatalyst, nanomaterials, metal oxide
\end{abstract}

\section{Introduction}

In the past years, semiconductor, used as photocatalytic materials in wastewater treatment and degradation of some toxic organic pollutants, has been extensively investigated [1-3]. Recently, however, nanosicence and nanotechnology have developed rapidly and almost have been involved in all the areas of production including catalyst field. So, considerable researches have been carried on in the area of nano-photocatalyst [4, 5]. Nano-photocatalysts, including nanoparticles and nanofibers, have seen tremendous research.

Nanoparticles show very high photocatalytic activity due to their high surface-tovolume ratio and high surface activity. However, the specific structure of nanoparticles also brings some shortages in the applications: due to the high surface activity, nanoparticles have a strong tendency to agglomerate into large particles which leads to the decrease of photocatalytic activity. So a good dispersion needs to be realized. And in addition, fine powdered nanoparticles are very difficult to be separated from the solution after the termination of photocatalytic reactions. Thus, as for the nano-photocatalyst, one of the most critical issues is how to make it well dispersed and at the same time easy to be recycled. Hence, researchers have been searching for reasonable solutions to realize the good dispersion and easy recycle of nano-photocatalyst, among which electrospinning method has been proved to be an effective one (Kim et al. [6]). 
In addition, electrospinning has also been playing a vital role in fabrication of photocatalytic nanofibers $[7,8]$. Compared with nanoparticles, nanofibers can be easily reclaimed from solution due to their 1D dimensional structure. Large scale area of nanofibers with photocatalytic activity can be obtained by electrospinning with very simple methods. Take metal oxide nanofibers for example, they are usually prepared by electrospinning the homogenous mixed solution of metal salt and polymer, the subsequent calcination decompose the polymer and turn the metal salt into metal oxide nanofibers. Many researchers have focused their attention on the photocatalytic activity of nanofibers $[9,10]$.

This review has summarized the recent researches and the important fundamental work in this area with an emphasis on the applications of electrospinning on nanophotocatalytic studies.

\section{Electrospinning applications on the nano-photocatalyst}

\section{Photocatalytic nanofibers fabrication by electrospinning}

$\mathrm{TiO}_{2}$ is the most commonly used semiconductor photocatalyst due to their stability, compatibility and non-toxic properties. So $\mathrm{TiO}_{2}$ nanofibers have seen tremendous research. $\mathrm{ZnO}$ is another kind of semiconductor which has been used as catalyst in many photocatalytic degradations. In many degradation reactions, $\mathrm{ZnO}$ has an analogous performance as $\mathrm{TiO}_{2}$. In the photocatalytic degradation of Rhodamine $\mathrm{B}$ $(\mathrm{RhB})$, for example, $\mathrm{ZnO}$ nanofibers have a stronger photocatalytic activity than $\mathrm{ZnO}$ nanoparticles. And this may be contributed to the porous structure of $\mathrm{ZnO}$ nanofibers, which could supply a higher contacting surface area between the catalyst and the target molecules and at the same time could hinder the agglomeration. Many other semiconductors, such as ZnS and CdS, have also attracted many studies (Zhou et al [11]).

Electrospinning is one of simple and versatile methods for generating photocatalytic nanofibers by combination with the sol-gel technique. Furthermore, during the electrospinning process, special structures can be fabricated by adjusting the technology parameters (Sun et al. [12]). Take photocatalytic $\mathrm{TiO}_{2}$ nanofibers preparation for example, $\mathrm{TiO}_{2}$ nanofibers composed of different crystals could be produced by controlling the calcination temperature. $\mathrm{TiO}_{2}$ has three crystal states: brookite, anatase and rutile. It was reported that $\mathrm{TiO}_{2}$ composed of anatase phase with a trace percentage of rutile phase had a better photocatalytic activity ( $\mathrm{Li}$ et al. [13]). During the calcination process, with the increase of the temperature $\mathrm{TiO}_{2}$ transfers from the anatase to the rutile witch can be explained by the phase transformation theory (Madhugiri et al. [14]). The Ti-O bond breaks in the anatase phase and rearranges in octahedral conformation to form the rutile phase. So the $\mathrm{TiO}_{2}$ nanofibers fabricated at different calcination temperatures exhibit different photocatalytic activity. By controlling the calcination temperature, the $\mathrm{TiO}_{2}$ nanofibers contained anatase phase with a trace percentage of rutile phase could be obtained. In addition, high Brunauer-Emmett-Teller (BET) surface area was retained. However, the anatase/rutile ratio is difficult to control utilizing this approach, so some strategies have been tried, among which electrospinning via hydrothermal method has been proved to be effective. During the process the percentage of anatase/rutile phase can be controlled by adjusting the hydrothermal time. 
Our research group has successfully prepared a series of inorganic metal oxide nanofibers by sol-gel process with electrospinning technique, including $\mathrm{TiO}_{2} / \mathrm{ZnO} / \mathrm{Mn}_{2} \mathrm{O}_{3} / \mathrm{BaO}$ nanofibers and so on.

Polyvinglpyrrolidone (PVP) was dissolved in the anhydrous ethanol to form the homogenous solution, and then the mixed solution of acetic acid (HAc) and titanium tetra-n-butoxide $\left(\mathrm{TiO}(\mathrm{Bu})_{4}\right)$ was added into the PVP solution to form the electrospun solution. The $\mathrm{PVP} / \mathrm{TiO}_{2}$ composite nanofibers were obtained by electrospinning and the subsequent calcination remove the polymer and produce the $\mathrm{TiO}_{2}$ nanofibers ( $\mathrm{Li}$ et al. [15]) (Shown in Fig.1a-b). The photocatalytic experiment indicates the same result as described above: the $\mathrm{TiO}_{2}$ nanofibers composed of anatase phase with a little percentage of rutile phase have better photocatalytic activity. With the analogous method, other metal oxide nanofibers, including $\mathrm{ZnO}, \mathrm{TiO}_{2} / \mathrm{ZnO}, \mathrm{ZnO} / \mathrm{BaO}$ and $\mathrm{ZnMn}_{2} \mathrm{O}_{4}$ nanofibers are all fabricated.

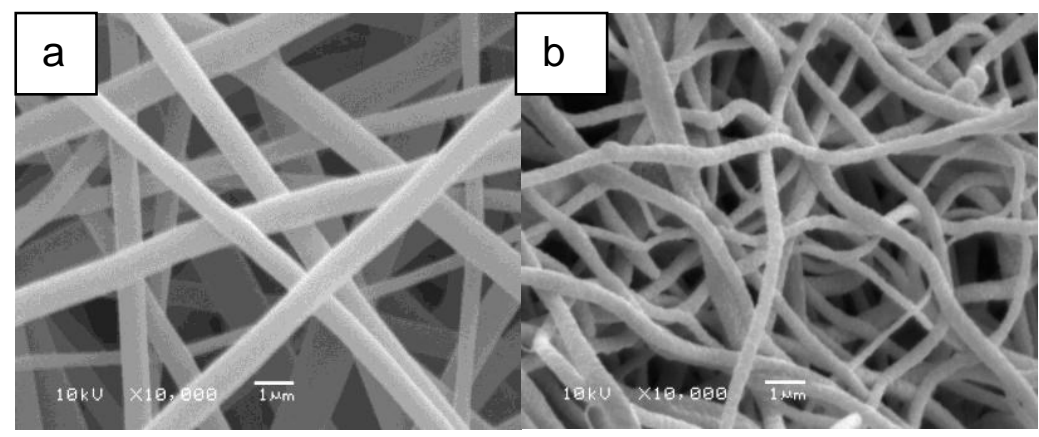

Fig. 1. SEM images of a) precalcined $\mathrm{PVP} / \mathrm{TiO}{ }_{2}$ composite nanofibers and b) $\mathrm{TiO}_{2}$ nanofibers after the heating process.

A novel method has also been developed in our lab to fabricate photocatalytic $\mathrm{TiO}_{2}$ nanofibers (Yin et al. [16]). Different from the usual method, our group used tetrabutyl titanate, ethanol and hydrochloric acid as reagent to produced $\mathrm{TiO}_{2}$ nanofibers by direct electrospinning method without polymer additives. $\mathrm{TiO}_{2}$ nanofibers with different morphology can be obtained through adjusting the ratio of the reagents. Fig. 2 shows the as-spun $\mathrm{TiO}_{2}$ nanofibers fabricated with direct electrospinning method. It can be observed that $\mathrm{TiO}_{2}$ nanofibers fabricated in this approach have a regular morphology with a smooth surface.

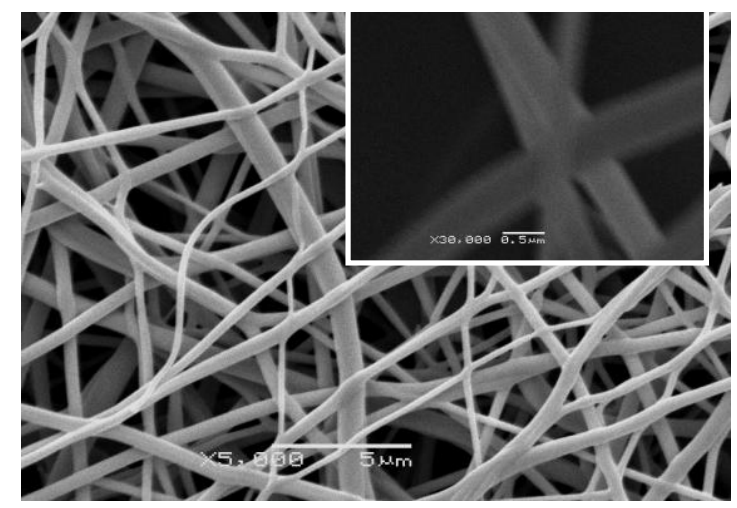

Fig. 2. SEM images of as-spun $\mathrm{TiO}_{2}$ fibers using direct electrospinning method. 
The decomposition of phenol was used to assess the photocatalytic activity of fabricated $\mathrm{TiO}_{2}$ nanofibers calcined at $550{ }^{\circ} \mathrm{C}$. The experimental setup consisted of a small reaction cell with the $\mathrm{TiO}_{2}$ nanofibers submerged in a $50 \mathrm{ppm}$ phenol solution. The UV lamps have an intensity of about $0.6 \mathrm{KW} / \mathrm{m}^{2}$ for the $360 \mathrm{~nm}$ excitation line at the position above the sample in distance of $30 \mathrm{~cm}$. The experiment was performed at the room temperature. The spectra of photocatalytical degradation of phenol on $\mathrm{TiO}_{2}$ nanofibers is shown in Fig. 3. After two hours, the degradation efficiency reached to $85 \%$, indicating that the fabricated $\mathrm{TiO}_{2}$ nanofibers have an excellent photocatalytic activity.

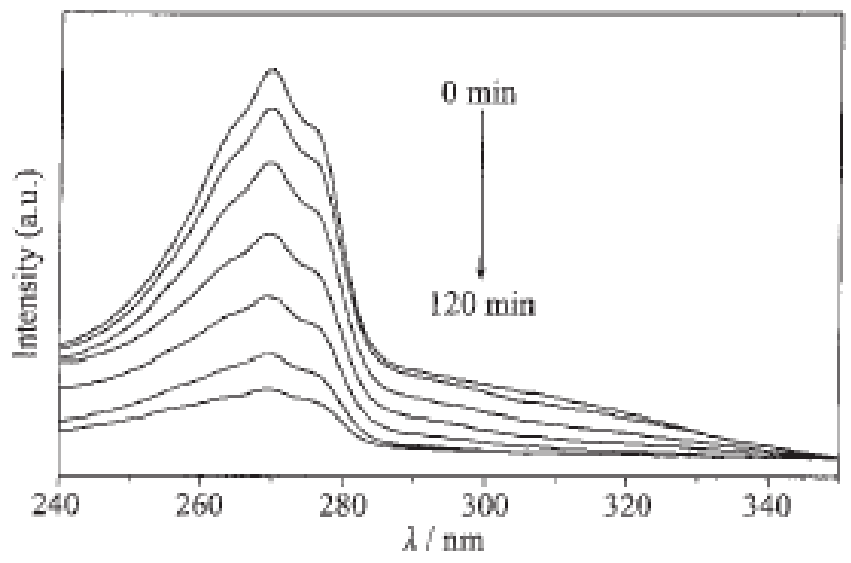

Fig. 3. Spectra of photocatalytic degradation of phenol on $\mathrm{TiO}_{2}$ nanofibers under the UV light irradiation, e.g .[15]

Except the nanofibers that have been identified to have photocatalytic activity such as $\mathrm{TiO}_{2}$ and $\mathrm{ZnO}$, many other organic nanostructures have been obtained in our lab. The preparation method and the properties that have been studied may supply some fundamental work for the development of nano-photocatalyst.

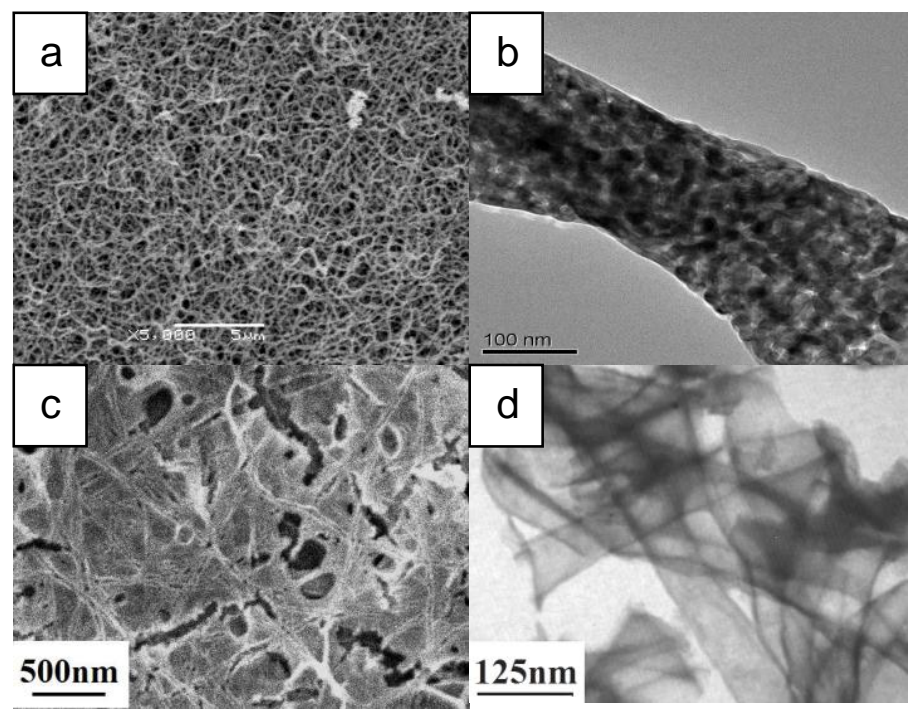

Fig. 4. SEM and TEM imagines of $(a, b) \mathrm{BaFe}_{12} \mathrm{O}_{19}$ nanofibers and (c, d) $\mathrm{ZrO}_{2}$ nanotubes. 
Ferrite nanofibers and some metal oxide nanotubes, for example, which might perform in nano-photocatalyst separation, were prepared by electrospinning via solgel technique [17, 18] (shown in Fig. 4).

\section{Composite photocatalytic nanofibers fabricated by electrospinning}

Many research have reported that, composite nanoparticles, such as $\mathrm{SnO}_{2} / \mathrm{TiO}_{2}$, $\mathrm{ZnO} / \mathrm{TiO}_{2}, \mathrm{ZnO} / \mathrm{SnO}_{2}$, have a higher photocatalytic activity than single nanoparticles (Liu et al. [19]). As for one certain kind nanoparticle, addition of another can reduce its bandgap, extend its adsorption region to the visible light, promote the electronhole separation under irradiation and achieve a higher photocatalytic activity. Take $\mathrm{TiO}_{2} / \mathrm{ZnO}$ composite nanomaterials for example, the band gap of $\mathrm{TiO}_{2}$ is $3.0 \mathrm{ev}$ for rutile and $3.2 \mathrm{ev}$ for anatase and that of $\mathrm{ZnO}$ is $3.5 \mathrm{ev}$. The ultraviolet (UV) light $(\lambda=290 \sim 400 \mathrm{~nm})$ can be divided into UVA $(\lambda=320 \sim 400 \mathrm{~nm})$ region and UVB $(\lambda=290 \sim 320 \mathrm{~nm})$ region, the UVB absorbance of bulk $\mathrm{TiO}_{2}$ is four times higher than that of $\mathrm{ZnO}$, but $\mathrm{TiO}_{2}$ does not absorb well through most of the UVA region. The addition of $\mathrm{ZnO}$ in $\mathrm{TiO}_{2}$ nanomaterials can enhance the UVA absorbing ability (David et al. [20]).

Many strategies, wet chemistry method (Wang et al. [21]) and coprecipitation method (Wang et al. [22]), for example, have been applied to synthesize composite photocatalytic nanomaterials. And in recent studies, electrospinning combined with hydrothermal technique has been identified to be an effective method for the preparation of composite nanomaterials, and in addition, compared with other methods, the nano-materials thus prepared were composed of nanofibers rather than nanoparticles, which can be recycled easily. $\mathrm{ZnO} / \mathrm{SnO}_{2}[23,24]$ nanostructures- $\mathrm{TiO}_{2}$ nanofibers heterostructures have been successfully synthesized.

\section{Nanoparticles dispersion via electrospinning}

It is well known that one of the most critical issues on the application of nanophotocatalysts is the dispersion. Many strategies have been tried to make a better dispersion. Electrospun nonwoven nanofibers can be used as a good support for photocatalysts so as to make a better dispersion.

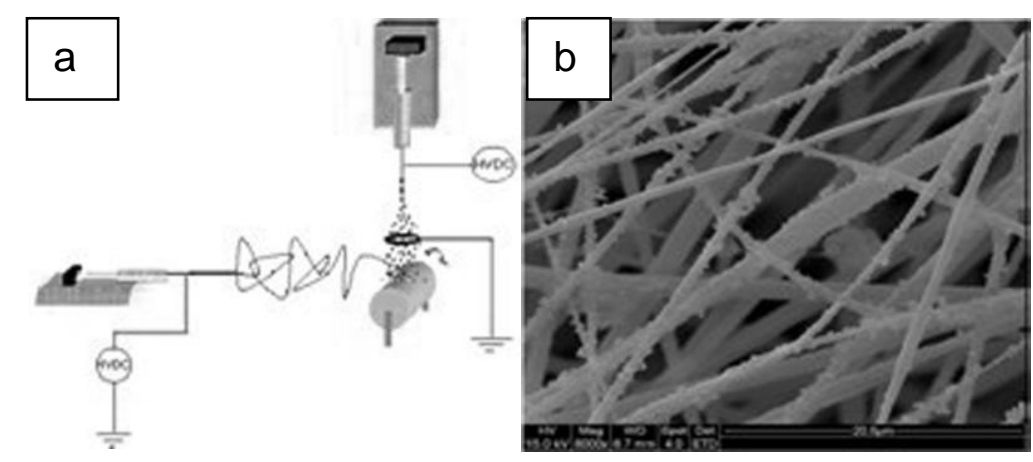

Fig. 5. Schematic diagram of a) electrospinning combined with electrospraying apparatus and SEM of b) obtained PSU nanofibers loaded $\mathrm{TiO}_{2}$ nanoparticles, e.g [25].

The specific large surface area of nanofibers can provide a holder to load nanoparticles to avoid the agglomeration. In addition, the porous structure of 
nanofibrous mats could supply a higher surface area, thus facilitates the reaction/interaction between the fibers and the interacting media, so high photocatalytic activity is retained. At the same time, due to the one dimensional structure, the recycling is easy to be achieved. Combined with electrospinning method, photocatalyst can be easily dispersed on the surface of some unique nanofibers. Coalescence of electrospinning with electrospraying is a successful method that has been practiced (Rose et al. [25]). The apparatus and the obtained nanofibers loaded $\mathrm{TiO}_{2}$ nanoparticles are shown in Fig. 5. Used polysulfone (PSU) as electrospun polymer, methanol as suspension solution, 3 (trimethoxysilyl) propyl methacylate as the suspension dispersant, electrospinning and electrospraying technique were applied at the same time. Seen from Fig. 5, $\mathrm{TiO}_{2}$ nanoparticles were uniformly distributed on the surface of PSU nanofibers. So the agglomeration was hindered. In addition, due to the one dimensional structure of PSU nanofibers, $\mathrm{TiO}_{2}$ nanoparticles could be easily reclaimed.

\section{Fabrication of $\mathrm{TiO}_{2}$ nanofibers coated with metal particles via electrospinning}

Many metal particles, Platinum (Pt) for example, have received considerable interest due to their excellent performance in catalytic activity, such as hydrogenation. On the basis of electrospinning, Pt deposited on the polymer or carbon nanofibers could have better performance (Graeser et al. [26]). It has been reported that it is possible to use Pt nanoparticles deposited on the anatase nanofibers as seeds to grow Pt nanowires (Lee et al. [27]). Some research have been carried on to deposited nanoparticles on anatase $\mathrm{TiO}_{2}$ nanofibers and moreover to fabricate Pt nanowires (Formo et al. [28]), as shown in Fig. 6. Pt nanowires are composed of $\mathrm{Pt}$ nanoparticles, so the active surface area is further enlarged, resulting in the higher catalytic activity.
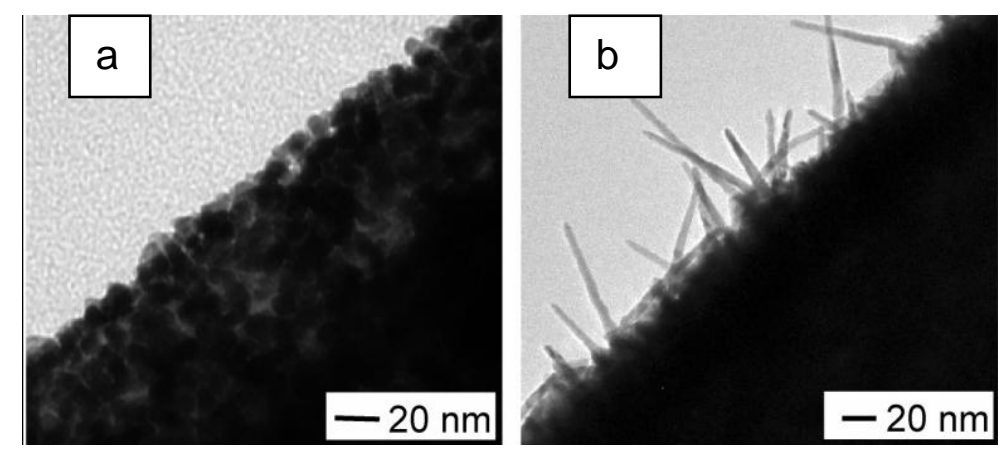

Fig. 6. TEM images of anatase $\mathrm{TiO}_{2}$ decorated with a) $\mathrm{Pt}$ nanoparticles and b) $\mathrm{Pt}$ nanowires, e.g.[26].

However, the deficiency of this research lies in ignoring the photocatalytic activity of $\mathrm{TiO}_{2}$ nanofibers. Here, $\mathrm{TiO}_{2}$ nanofibers act just as a supporter for the Pt catalyst. In fact, $\mathrm{TiO}_{2}$ nanofibers also have photocatalytic activity. So the mutual effect of photocatalytic $\mathrm{TiO}_{2}$ nanofibers and $\mathrm{Pt}$ nanowires should be investigated. It has been proved that Aluminum (III)-modified $\mathrm{TiO}_{2}$ has a far better photocatalytic activity than pure $\mathrm{TiO}_{2}$ in dye degradation (Zhao et al. [29]). The degradation of dyes under visible irradiation is initiated by the interfacial electron injection from the excited dye molecules to the $\mathrm{TiO}_{2}$ catalyst (Kyung et al. [30]). Because of the modification, an insulating thin $\mathrm{Al}_{2} \mathrm{O}_{3}$ layer is formed between the dyes and $\mathrm{TiO}_{2}$ catalyst, which acts as a barrier and thus inhibits the electron back transfer from the catalyst to the dye 
cations, resulting in a higher photocatalytic activity. Other metal modified $\mathrm{TiO}_{2}$ also performed enhanced photocatalytic activity compared with single $\mathrm{TiO}_{2}$ catalyst (Wang et al. [31]).

So it can be assumed that the immobilization of $\mathrm{Pt}$ nanoparticles on the anatase nanofibers surface could influence the photocatalytic activity of $\mathrm{TiO}_{2}$. If the existence of $\mathrm{Pt}$ could improve the photoactivity of $\mathrm{TiO}_{2}$, the $\mathrm{TiO}_{2}$ nanofibers loaded with $\mathrm{Pt}$ nanoparticles can be served as catalyst in a larger catalytic area, dye pollutants degradation, hydrogenation and so on.

\section{Preparation of photocatalytic nanostructures via Atom Layer Deposition (ALD) method based on electrospinning}

Atom Layer Deposition (ALD) is a straightforward method to deposit conformal thin films of many materials, including metals, oxides, nitrides, fluorides and so on. Combined with the template method, different structural materials can be fabricated by ALD [32, 33]. Electrospinning combined with ALD, as a technique to fabricate nanomaterials with different structures, has attracted much attention. Nanofibers fabricated by electrospinning are composed of organic polymer or a mixture of organic polymer and inorganic precursor. If the former is coated by ALD, nanotubes can be obtained. For the latter, coaxial nanofibers can be fabricated.

Utilizing electrospinning method combined with ALD technique, novel magnetic and photocatalytic composite nanomaterials are successfully fabricated (Santala et al. [34]). $\mathrm{CoFe}_{2} \mathrm{O}_{4}-\mathrm{PVP}$ nanofibers were first prepared, and then were coated with $\mathrm{TiO} 4$ by ALD. The subsequent calcinations resulted in the formation of $\mathrm{TiO}_{2}$ nanotubes loaded $\mathrm{CoFe}_{2} \mathrm{O}_{4}$. Due to the magnetic properties of $\mathrm{CoFe}_{2} \mathrm{O}_{4}$, this kind of photocatalysts is very easy to reclaim.

Layer-by-layer (LBL) method, which involves the sequential adsorption of opposite charge materials to construct ultrathin conformal coatings, has a similar procedure with ALD (Lee et al. [35]). The difference is that LBL method is always used to produce multi-layer materials. $\mathrm{TiO}_{2}$ nanotubes loaded $\mathrm{CoFe}_{2} \mathrm{O}_{4}$ in inner and nanofibers coated with $\mathrm{TiO}_{2}$ nanoparticles are shown in Fig. 7 .

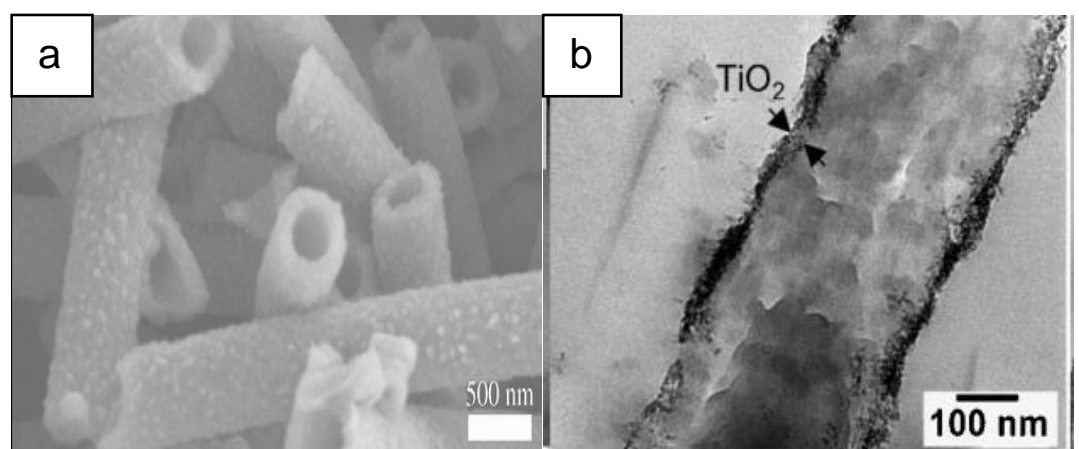

Fig. 7. SEM a) image of $\mathrm{TiO}_{2}$ nanotubes loaded $\mathrm{CoFe}_{2} \mathrm{O}_{4}$ in inner and $\mathrm{TEM}$ b) image of nanofibers coated with $\mathrm{TiO}_{2}$ nanoparticles, e.g [34, 35].

\section{Conclusions}

As an environmentally friendly technical which could offers the potential for a complete elimination of toxic chemicals in the environmental through its efficiency 
and broad applicability, photocatalyst has attracted abundant attention in many areas. And in the future, the worldwide emphasized notice in circumstance will make it attract more and more attention. Nano-photocatalyst, due to its special structure, will be a very important embranchment in this area. Electrospinning, as a versatile and simple method to fabricate nano-materials, will still play an important role in nano-photocatalyst development. New kind nano-photocatalyst, composite nanoparticles for example, and new fabrication methods, electrospinning via hydrothermal method mentioned above for instance will be probably the research direction.

\section{Acknowledgements}

This study was partly supported by the Beijing Natural Science Foundation, PHR (IHLB), the 863 Project (grant no. 2007 AA021906) and the 973 Project (grant no. 2010CB 933501).

\section{References}

[1] Li, D.; Dong, W.; Sun, S.; Shi, Z.; Feng, S. J. Phys. Chem. C. 2008, 112, 14878.

[2] Zhao, W.; Sun, Y.; Castellano, F. J. Am. Chem. Soc. 2008, 130, 12566.

[3] Wang, Q.; Chen, C.; Zhao, D.; Ma, W.; Zhao, J. Langmuir. 2008, 24, 7338.

[4] Li, J.; Ma, W.; Chen, C.; Zhao, J.; Zhu, H.; Gao, X. J. Mol. Catal. A: Chem 2007, 261,131 .

[5] Tian, M.; Wu, G.; Adams, B.; Wen, J.; Chen, A. J. Phys. Chem. C. 2008, 112, 825.

[6] Kim, S.; Lim, S. K. Appl. Catal. B. 2008, 84,16.

[7] Uyar, T.; Kingshott, P.; Besenbacher, F. Angew. Chem. Int. Ed. 2008, 47, 9108.

[8] Singh, G.; Bittmer, A. M.; Loscher, S.; Malinowski, N.; Kern, K. Adv. Mater. 2008, 20, 2332.

[9] Chuangchote, S.; Jirputti, J.; Sugawa, J.; Kawa, S. Y.; Applied. Materials. Omterfaces. 2009, 1, 1140.

[10] Alres, A. K.; Berutti, F. A.; Clemens, F. G.; Granle, T.; Bergmann, C. P. Mater. Res. Bull. 2009, 44, 312.

[11] Zhou, Z.; Feng, Y.; Xu, W.; Ren, F.; Ma, H. J. Appl. Polym. Sci. 2009, 113,1264. [12]Sun, C.; Wang, N.; Zhun, S.; Hu, X.; Zhou, S.; Chen, P.; Chem. Commun. 2008, 3293.

[13] Li, G. H.; Gray, K. A. Chem. Phys. 2007, 339, 173.

[14] Madhugiri, S.; Sun, B.; Smirniotis, P. G.; Ferraris, J. P.; Balkns, K. J.; Mircroporous. Mesoporous. Materials. 2004, 69, 77.

[15] Li, C.; Zhao, G.; Fu, Z.; Wang, P.; Chang, M.; Li, X. Chinese j. inorg. chem. 2006, 22, 2061.

[16] Yin, L.; Li, C.; Fu, J.; Li, X. Nanomats preparation and fabrication method by electrospinning. CN Patent, 2008, 101284226.

[17] Guo, Y.; Li, C.; Wang, J. Chinese J. Inorg. Chem. 2009, 25, 1018.

[18] San, Z.; Li C.; Li, X. Chinese J. Inorg. Chem. 2007, 23, 879.

[19] Liu, H.; Yang, J.; Liang, J.; Huang, Y.; Tang, C. J. Am. Ceram. Soc. 2008, 91, 287.

[20] David, M. K.; Liang, X. H.; Casey, S. C.; Luis, F. H.; Li, P.; Alan, W. W. Adv. Funct. Mater. 2008, 18, 607.

[21] Wang, X. W.; Liu, G.; Chen, Z. G.; Li, F.; Wang, L. Z.; Lu, G. Q.; Chen, H. M.; Chem. Commun. 2009, 3452. 
[22] Wang, Z. Y.; Huang, B. B.; Dai, Y.; Qin, X. Y.; Zhang, X. Y.; Wang, P.; Liu, H. X.; Yu, J. X. J.Phys.Chem.C. 2009, 113, 4612.

[23] Wang, N. X.; Sun, C. H.; Zhao, Y.; Zhao, S. Y.; Chen, P.; Jiang, L. J. Mater. Chem. 2008, 28, 3909.

[24] Wang, C. H.; Shao, C. L.; Zhang, X. T.; Liu, Y. C. Inorg. Chem. 2009, 48, 7261. [25] Rose, M.; Sundarrajan, S.; Pliszka, D.; Ramakrishna, S.; Modestil, M. Nanotechnology 2008, 19, 285707.

[26]Graeser, M.; Pippel, E.; Greiner, A.; Wendorff, J. Macromolecules. 2007, 40, 6032.

[27] Lee, E. P.; Chen, J.; Yin, Y.; Campbell, C. T.; Xia, Y. Adv. Mater. 2006, 18, 3271. [28] Formo, E.; Lee, E.; Campbell, D.; Xia, Y. Nano. Lett. 2008, 8, 668.

[29] Zhao, D.; Chen, C.; Wang, Y.; Ma, W.; Zhao, J.; Rajh, T.; Zang, L.; Environ. Sci. Technol. 2008, 42, 308.

[30] Kyung, H.; Lee, J.; Choi, W.; Sci. Technol. 2005, 39, 2376.

[31] Wang, Y.; Zhao, D.; Ma, W.; Chen, C.; Zhao, J.; Environ. Sci. Technol. 2008, 42, 6173.

[32] Bachmann, J.; Jing, J.; Knez, M.; Barth, B.; Shen, H.; Mathur, S.; Sele, U.G.; Nielsch, K.; J. Am. Chem. Soc. 2007, 129, 9554.

[33] Robin, H. A.; Marianna, K. M.; Wit, J. D.; Ritala, M.; Brinke, G. T.; Leskelä, M.; Ikkala, O.; Adv. Mater. 2007, 19, 102.

[34] Santala, E.; Kemell, M.; Leskela, M.; Ritala, M.; Nanotechnology. 2009, 20 , 035602.

[35] Lee, J. A.; Krogman, K. C.; Ma, M.; Hill, R. M.; Hammond, P. T.; Rutledge, G. C. Adv. Mater. 2009, 21, 1252. 